\title{
In Situ Measurements of Interstellar Dust
}

\author{
M. Landgraf and E. Grün \\ Max-Planck-Institut für Kernphysik \\ D-69029 Heidelberg, Germany
}

\begin{abstract}
We present the mass distribution of interstellar grains measured in situ by the Galileo and Ulysses spaceprobes as cumulative flux. The derived in situ mass distribution per logarithmic size interval is compared to the distribution determined by fitting extinction measurements. Large grains measured in situ contribute significantly to the overall mass of dust in the local interstellar cloud. The problem of a dust-to-gas mass ratio that contradicts cosmic abundances is discussed.
\end{abstract}

\section{Introduction}

Dust alters the conditions of the diffuse medium, e.g. it provides a dominant heating mechanism via photo-electric heating (Slavin \& Frisch, 1997). So understanding the size distribution is important for modeling the diffuse interstellar medium of which the local bubble is an example. Extinction measurements give information about the size distribution of grains in the diffuse medium and hints at their composition (Massa \& Savage, 1989). The extinction curve can be fitted by a mixture of graphite and silicate grains with a power-law size distribution (MRN distribution) $n(a) \propto a^{-q}$ in the interval $0.005 \mu \mathrm{m} \leq a \leq 0.25 \mu \mathrm{m}$ with an exponent of $3.3 \leq q \leq 3.6$ (Mathis et al. , 1977). This optically active population can explain all depletions (Spitzer, 1978). No larger grains, that are optically gray, are needed. Interstellar dust was first detected in situ by the dust detector on board the spaceprobe Ulysses in 1992 after Jupiter flyby (Grün et al. , 1993). The detector, that is identical to the one on board the Galileo spaceprobe, is an impact charge detector and measures the mass, impact velocity, and impact direction of the impacting grain as described by Grün et al. (1992). The finding was confirmed by the data collected by the Galileo dust detector. Galileo reached Jupiter in December 1995 where planetary dust is dominant, so the Galileo measurements range from mid 1993, when Galileo left the inner solar system, to the end of 1995. Ulysses dust data is available up to March 1996. The criteria to identify interstellar impacts in both Ulysses and Galileo data were given by Baguhl et al. (1996). The impact rate measured by Ulysses is 0.45 day $^{-1}$ which translates into a flux of $1 \cdot 10^{-4} \mathrm{~m}^{-2} \mathrm{~s}^{-1}$. Interstellar grains provide the dominant dust flux in the outer solar system.

Analysis of the directional information (Baguhl et al. , 1995) indicates a stream of grains entering the solar system from a direction that is compatible with the upstream direction of interstellar helium (Witte et al. , 1993). 


\section{Fit to Cumulative Mass Distribution}

The grains detected in situ are larger than the ones needed to explain the extinction curve. We check if the mass distribution is just an extrapolation of the MRN population, ignoring the fact that this would contradict cosmic abundance considerations (Spitzer, 1978). To avoid binning effects, we investigate the cumulative mass distribution of flux $F(m)$. This is the flux of grains with masses larger than a given mass.

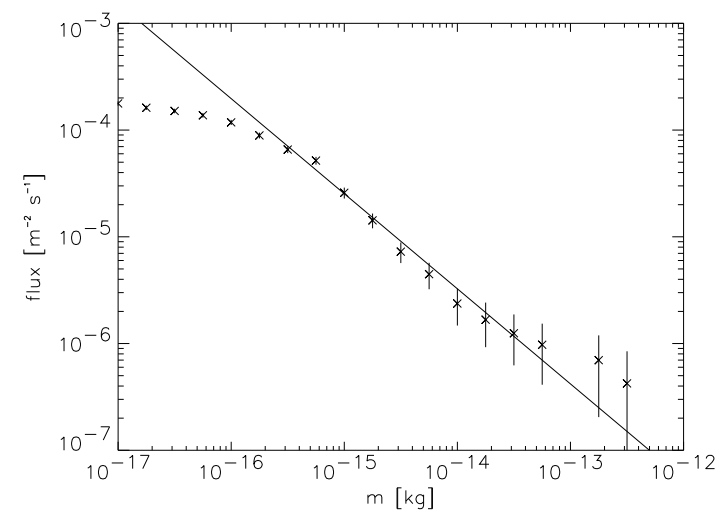

Fig. 1. Cumulative mass distribution of grains detected by Ulysses and Galileo including power-law fit. Error-bars indicate statistical errors.

For the MRN distribution we expect $F_{\mathrm{MRN}}(m) \propto m^{-q^{\prime}}$ with $q^{\prime}=0.83$. Fig.1 shows the cumulative mass distribution of all particles detected by Ulysses and Galileo together with the power-law fit to masses larger than $10^{-16} \mathrm{~kg}$, where the distribution was not altered by filtration as described by Levy and Jokipii (1976). The fit is weighted to match the points with good statistics. An exponent of $q^{\prime}=0.90$ fits the data best with $\chi^{2}=9.4 . \chi^{2}$ is well below the $1 \sigma$-limit of $\chi_{1 \sigma, 12}^{2}=14$ for 12 degrees of freedom. Due to bad statistics for grains with large masses, the fit is not too sensitive, but indicates a steeper slope than the MRN extrapolation. The $\chi^{2}$ for the MRN value is close to the $1 \sigma$-limit $\chi_{\mathrm{MRN}}^{2}=14$. Slopes of $q^{\prime}>1$ are ruled out on the $1 \sigma$-level with $\chi_{q^{\prime}>1}^{2}=20$. Such a steep dropoff would not lead to a problem with cosmic abundances when extrapolating to large masses since the small grains would then contribute more to the overall mass than the large ones.

\section{Comparison to Extinction Data}

We compare the in situ distribution to the distribution fitted to extinction measurements by Kim et al. (1994). Since extinction is measured along long lines of 
(a)

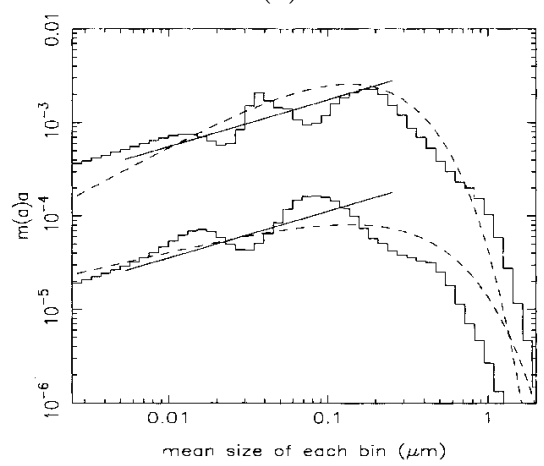

(b)

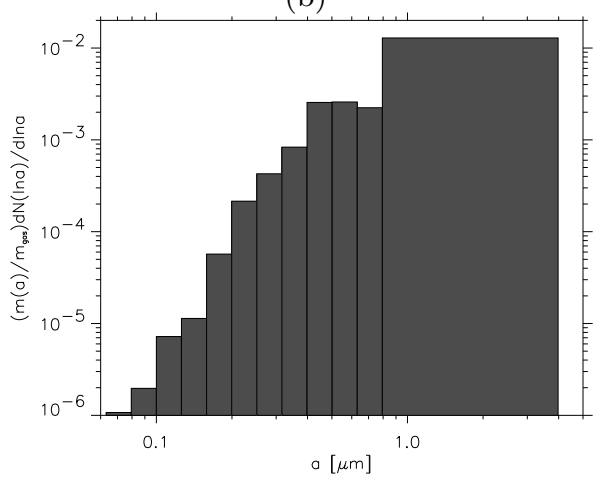

Fig. 2. (a) Differential distribution of mass in silicate (upper histogram) and graphite grains (lower histogram, scaled down by a factor 10) per logarithmic size interval normalized to the mass of hydrogen in the same volume (taken from (Kim. et al. , 1994), the abscissa is the mean grain size in the bin and the ordinate is identical to the ordinate in (b).). The solid line is the MRN distribution and the dashed line is the PED(Power-law with Exponential Decay) fit. (b) Mass distribution per logarithmic size interval of in situ data.

sight through the diffuse medium and in situ measurements are very local compared to this, one has to be careful interpreting this comparison. The fits to extinction measurements rely on a dust model with a size distribution and different grain compositions. Kim et al. (1994) give the mass per logarithmic size interval normalized to the mass of hydrogen in the same volume as shown in Fig.2 (a) assuming the canonical value of $0.1 \mathrm{H}$-atoms $\mathrm{cm}^{-3}$ for the gas density in the diffuse medium. We calculate the size distribution $N(\ln a)$ from the mass distribution given in Sect.2 by assuming homogeneous, spherical grains with a bulk density of $\rho_{\text {ISP }}=2.5 \mathrm{~g} \mathrm{~cm}^{-3}$ (Burns et al. , 1979). From the size distribution the differential mass distribution per logarithmic size interval $\left(m(a) / m_{\text {gas }}\right) \mathrm{d} N(\ln a) / \mathrm{d} \ln a$, which is identical to the function given by Kim et al. is calculated. The comparison of Fig.2 (a) with Fig.2 (b) shows, that the in situ distribution does not reproduce the steep dropoff fitted to the extinction measurements. The in situ distributions show, that there is much mass in grains larger than $0.2 \mu \mathrm{m}$.

\section{Discussion}

We have shown that the existence of big $(a>0.2 \mu \mathrm{m})$ interstellar grains is evident. If the size distribution of small grains is extrapolated to larger sizes as was indicated by Fig.2 (b), this will lead to dust-to-gas mass ratios of more than the canonical value of $1 \%$, because $1 \%$ is already used up by MRN grains 
and larger grains contribute even more to the overall mass assuming the MRN extrapolation. Dust-to-gas mass ratios larger than $1 \%$ contradict cosmic abundances (Spitzer, 1978). By integration of the distribution shown in Fig.2 (b), the contribution of the in situ measured particles to the dust-to-gas mass ratio is determined to be $2.2 \%$. The problem of too much mass in dust in the VLISM gets even worse if one extrapolates the size distribution to even larger interstellar grains, which were identified recently by Taylor et al. (1996) in radar meteor data. Of course the extrapolation of a MRN-like power-law is limited otherwise the total mass in dust gets infinite.

Jones et al. (1996) show that large grains get destroyed in shocks caused by supernovae. If the dust in the LIC has been shocked in the past, than there should be much more smaller grains, and if not, the dust has to be younger than the typical time-scale for returning the dust mass into the gas phase by supernova shocks which is $\tau_{\mathrm{SNR}} \approx 10^{8}$ a (Jones et al. , 1996).

\section{References}

Baguhl M., Hamilton D.P., Dermott S.F., et al. 1995 Science, 268, 1016

Baguhl M., Grün E., \& Landgraf M. 1996 Page 165 of: von Steiger, R., Lallement, R., \& Lee, M.A. (eds), Space Sci. Rev., 78, Kluwer Academic Publishers

Burns J.A., Lamy P.L., \& Soter S. 1979 Icarus, 40, 1

Grün E., Fechtig H., Hanner M.S., Kissel J., Lindblad B.-A., Linkert D., Maas D., Morfill G.E., \& Zook H.A. 1992 Space Sci. rev., 60, 317

Grün E., Zook H.A., Baguhl M., et al. 1993 Nature, 362, 428

Grün E., Baguhl M., Fechtig H., Kissel J., Linkert D., Linkert G., \& Riemann R. 1995a Planet. Space Sci., 43, 941

Grün E., Baguhl M., Divine N., et al. 1995b Planet. Space Sci., 43(8), 953

Grün E., Baguhl M., Divine N., et al. 1995c Planet. Space Sci., 43(8), 971

Jones A.P., Tielens A.G.G.M., \& Hollenbach D.J. 1996 Astrophys. J., 469, 740

Kim S.-H., Martin P.G., \& Hendry P.D. 1994 Astrophys. J., 422, 164

Levy E.H., \& Jokipii J.R. 1976 Nature, 264, 423

Massa D., \& Savage B.D. 1989 Page 3 of: Allamandola, L.J., \& Tielens, A.G.G.M. (eds), Interstellar dust IAU Symp., vol. 135, Kluwer Academic Publishers

Mathis J.S., Rumpl W., \& Nordsieck K.H. 1977 Astrophys. J., 280, 425

Slavin J., \& Frisch P. 1997 this volume

Spitzer L. 1978 Page 153 of: Physical Processes in the Interstellar Medium, New

York: Wiley Interscience

Taylor D., Baggaley W.J., \& Steel D.I. 1996 Nature, 380, 323

Witte M., Rosenbauer H., Banaszkiewicz H., \& Fahr H. 1993 Adv. Space Res., 13, (6) 121 